\title{
Does Illusory Flickering Result from Rhythmic Sampling of Visual Stimuli?
}

\author{
Maité Crespo-García ${ }^{1}$ and Thomas Hartmann ${ }^{2}$ \\ ${ }^{1}$ Department of Psychology, University of Konstanz, 78457 Konstanz, Germany, and ${ }^{2}$ Center for Mind/Brain Sciences, University of Trento, 38122 Trento, \\ Italy \\ Review of Sokoliuk and VanRullen
}

Approximately a century ago, the oscillatory character of human neural activity was revealed by the discovery of electroencephalographic (EEG) rhythms. Since then, numerous observations have helped to decipher the functional meaning of brain oscillations. Most of the studies have focused on the alpha rhythm $(8-13$ $\mathrm{Hz}$ ), whose strong spectral power is clearly modulated by visual stimulation, visuospatial attention, and working memory load. A robust finding is that alpha power decreases over engaged visual regions (e.g., contralateral to the attended field), whereas alpha power increases over competing regions, pathways, or system modalities. Based on substantial evidence, modern frameworks propose that alpha power decreases reflect active information processing whereas alpha power enhancements reflect top-down inhibition of taskirrelevant regions (Klimesch et al., 2007).

On a global scale, alpha-mediated inhibition is an essential part of a gating mechanism by which some areas are disengaged, allowing the allocation of processing resources in task-relevant areas (Jensen and Mazaheri, 2010). On a more

Received Oct. 21, 2013; revised Nov. 21, 2013; accepted Nov. 25, 2013. M.C.-G. was supported by a grant from the Deutsches Forschungsgemeinschaft (DFG DA1485/1-1). T.H. was supported by a grant from the European Research Council (WIN2CON; ERC StG 283404). We thank Sarang S. Dalal for helpful discussions.

Correspondence should be addressed to Maité Crespo-García, Department of Psychology, University of Konstanz, 78457 Konstanz, Germany. E-mail:maite.crespo-garcia@uni-konstanz.de.

DOI:10.1523/JNEUROSCI.4486-13.2014

Copyright $\odot 2014$ the authors $\quad 0270-6474 / 14 / 340343-03 \$ 15.00 / 0$ local scale, it has an additional aspect: alpha oscillations exert a rhythmic modulation over the excitability of target neural populations (Klimesch et al., 2007). Through this oscillatory mechanism, neuronal activation is more likely at specific phases of the alpha wave, providing controlled timing for cortical processing and communication.

Numerous psychophysical reports are compatible with the idea that perception is a discrete process, intrinsically related to the timing of neuronal processes (for review, see VanRullen and Koch, 2003). Curiously, two successive visual events can be interpreted as simultaneous if they are presented within a temporal frame of around $100 \mathrm{~ms}$ (Lichtenstein, 1961), a period similar to the alpha cycle. Although a relationship between temporal framing in visual perception and alpha phase is still not clear, recent studies have shown that visual detection threshold fluctuates with the phase of ongoing alpha oscillations (Busch et al., 2009; VanRullen et al., 2011). In addition, cross-correlation analyses of visual stimulation and EEG sequences have shown that random visual flow causes an echo or reverberation of individual alpha oscillatory responses (VanRullen and Macdonald, 2012). Interestingly, removing the alpha frequency components from the stimulation sequence eliminates the EEG alpha echo.

Could we be aware of our pulsing perception imposed by the alpha rhythm? This question was addressed by Sokoliuk and VanRullen (2013). These authors introduced a static wheel stimulus based on MacKay rays (MacKay, 1957) that creates a visual illusion of having a regular and cyclic flickering on its center when perceived with the visual periphery. Sokoliuk and VanRullen (2013) propose that the flicker is experienced as a consequence of processing fluctuations caused by the alternation of favorable and unfavorable phases in the alpha wave. In other words, the flickering wheel illusion may allow conscious perception of cortical alpha oscillations. This proposal is based primarily on three observations. The most robust result Sokoliuk and VanRullen (2013) describe is a strong relationship between alpha power modulations and flicker perception. In a smooth pursuit experiment, participants fixated their gaze on a moving dot that revolved around a static, illusory wheel stimulus. The authors observed that the likelihood of flicker detection and occipital alpha power rose and fell together on each revolution of the moving dot. Additionally, the peak frequency of flicker-induced alpha modulations was significantly correlated with the individual alpha frequency. The authors suggest that the flicker illusion is not only the consequence of alpha oscillations, but can also enhance this activity through reverberation mechanisms (VanRullen and Macdonald, 2012).

It is not clear, however, whether alpha power enhancements occurred in regions processing the visual stimulus or in re- 
gions processing the task-irrelevant space. Curiously, perceiving the illusory flicker was more likely when participants fixated their gaze in the upper left quadrant of the screen, while the wheel stimulus remained centered on the screen. Although the reason for such non-uniform perception is not discussed by the authors, it might be related to the right-lateralized distribution of occipital alpha power, also observed in the case of the control stimulus. Previous studies have demonstrated that alpha synchronization occurs over areas processing the unattended space in a retinotopically organized pattern (Rihs et al., 2007). It is reasonable to suppose that although participants fixated their gaze on the dot, their spatial attention was directed to the visual stimulus, an effect likely enhanced by the perception of the flicker they had to report. Therefore, during maximal flicker perception, covert attention may have actually been oriented toward an inferior right position. In accordance with Rihs et al. (2007), the observed alpha power enhancements over right occipital sites are specific for this orientation of attention. Further analyses taking into account alpha power distributions at scalp or source level relative to gaze position would help to clarify this issue.

A psychophysical matching task provided further evidence of the relationship between alpha activity and the perception of the illusory flicker. Here, participants compared the illusory flickering with a reference stimulus that flickered artificially. The task consisted of regulating the frequency and intensity of the simulated flicker to make it match the illusory one. Interestingly, the average flicker frequency reported by the participants was in the alpha frequency band, around $9 \mathrm{~Hz}$. However, the total range of perceived frequencies was rather wide, from very low frequencies $(\sim 2 \mathrm{~Hz})$ to higher frequencies normally associated with the beta band $(20 \mathrm{~Hz})$. Although Sokoliuk and VanRullen (2013) attributed this variability to an inherent difficulty in perceiving temporal frequency with peripheral vision, other interpretations are also possible. As can be observed in the distribution of estimated frequencies (Sokoliuk and VanRullen, 2013, their Fig. 2 D), additional local maxima are evident at $\sim 5, \sim 15$, and possibly also at $\sim 20 \mathrm{~Hz}$. This distribution might suggest that participants were able to perceive not only the main flicker frequency, but also their harmonics, perhaps due to entrainment of underlying neuronal oscillators (Rager and Singer, 1998). Also, a purely behavioral response bias toward the fundamental and harmonic frequencies could explain the data. Therefore, the principal effect may actually have been represented by the $\sim 5 \mathrm{~Hz}$ response. Supporting this possibility, a recent study has shown that covert selective attention samples two simultaneous visual locations with antiphasic $4 \mathrm{~Hz}$ rhythms (Landau and Fries, 2012). In that study, the authors propose that there is an attentional sampling process following an ongoing endogenous rhythm of $\sim 8 \mathrm{~Hz}$. This means that each spatial location is sampled every $125 \mathrm{~ms}$, which could reconcile with the hypothesis of the visual temporal frame. In addition, it leaves open the question of whether the perceived flicker frequencies reported by Sokoliuk and VanRullen (2013) could be the result of a cortical mechanism based on theta $(4-8 \mathrm{~Hz})$ rather than alpha oscillations. Regardless of which specific rhythm is determining the flickering perception, the results are compatible with a phase-amplitude coupling mechanism by which the phase of slower oscillations governs attentional sampling through synchronization of gamma-band (40-100 Hz) networks involved with visual perception (Fries, 2009). However, the broad range of frequencies reported by the participants in the study by Sokoliuk and VanRullen (2013) casts a reasonable doubt on whether there is indeed a relationship between the perceived flickering frequency and alpha oscillations. To provide a stronger link between the phenomena, it would be helpful to demonstrate a strong correlation between reported flicker frequencies and individual alpha frequencies during maximal flicker perception.

Lastly, Sokoliuk and VanRullen (2013) tested whether the visual flickering illusion could be partly generated by eye movements. They addressed this question in two ways. First, they reanalyzed data from the smooth pursuit experiment after discarding time points with eye movements larger than $3^{\circ}$. Results were identical: the likelihood of perceiving the flicker was higher when alpha power increased. This approach does not fully eliminate the possibility that smaller eye movements such as tremors or microsaccades could contribute to the perception (or enhancement) of the visual illusion (Troncoso et al., 2008). However, in a further experiment, Sokoliuk and VanRullen (2013) demonstrated that the illusory flickering was also perceived in the afterimage. Therefore, small eye movements were not necessary to explain the illusory flicker, at least after its generation. Rather, it could depend on changes in the neural representations of motion processing circuitry that might be modulated by the alpha rhythm. The wheel illusion shares similar visual properties with other motion illusions (recently summarized in Ito, 2012): 1) it presents a radial pattern, 2) the flicker is better perceived with peripheral vision and enhanced by eye movements, and 3) contrast (Backus and Oruç, 2005) or spatial frequency (number of spokes of the wheel) strongly determine the illusory character of the stimulus (Sokoliuk and VanRullen, 2013). These similarities suggest that the flickering wheel illusion could affect neural substrates dedicated to the interpretation of motion signals, as happens with several motion illusions. For example, an illusory phenomenon called the motion aftereffect occurs when changing the gaze to a stationary scene after looking at a moving stimulus for an extended period of time. Although the physiological bases of the motion after effect are not completely understood, the evidence points to the adaptation of motion-sensitive neurons in different areas of the visual cortex, where the human motion area MT $+/ \mathrm{V} 5$ plays a special role (Hogendoorn and Verstraten, 2013). Whether slower oscillations synchronize the response of motion-sensitive neurons, potentially resulting in a flicker perception, would be an interesting question to analyze in future studies.

To conclude, the work of Sokoliuk and VanRullen (2013) revives the fascinating question about the lack of continuity in our vision, providing compelling evidence in support of a rhythmic sampling of visual perception. Further research should be carried out to firmly establish whether it is the cycle of alpha or perhaps another low-frequency oscillation that determines the illusory flicker for their stimulus. Finally, to fully characterize the oscillatory nature of perception, we must better understand the neural substrates that maintain this effect in the afterimage.

\section{References}

Backus BT, Oruç I (2005) Illusory motion from change over time in the response to contrast and luminance. J Vis 5:1055-1069. CrossRef Medline

Busch NA, Dubois J, VanRullen R (2009) The phase of ongoing EEG oscillations predicts visual perception. J Neurosci 29:7869-7876. CrossRef Medline

Fries P (2009) Neuronal gamma-band synchronization as a fundamental process in cortical computation. Annu Rev Neurosci 32:209224. CrossRef Medline

Hogendoorn H, Verstraten FA (2013) Decoding 
the motion aftereffect in human visual cortex. Neuroimage 82:426-432. CrossRef Medline

Ito $\mathrm{H}$ (2012) Illusory object motion in the centre of a radial pattern: the pursuit-pursuing illusion. Iperception 3:59-87. Medline

Jensen O, Mazaheri A (2010) Shaping functional architecture by oscillatory activity: gating by inhibition. Front Hum Neurosci 4:186. CrossRef Medline

Klimesch W, Sauseng P, Hanslmayr S (2007) EEG alpha oscillations: the inhibition-timing hypothesis. Brain Res Rev 53:63-88. CrossRef Medline

Landau AN, Fries P (2012) Attention samples stimuli rhythmically. Curr Biol 22:10001004. CrossRef Medline

Lichtenstein M (1961) Phenomenal simultane- ity with irregular timing of components of the visual stimulus. Percept Mot Skills 12:47-60. CrossRef

MacKay DM (1957) Moving visual images produced by regular stationary patterns. Nature 180:849-850. CrossRef

Rager G, Singer W (1998) The response of cat visual cortex to flicker stimuli of variable frequency. Eur J Neurosci 10:1856-1877. CrossRef Medline

Rihs TA, Michel CM, Thut G (2007) Mechanisms of selective inhibition in visual spatial attention are indexed by alpha-band EEG synchronization. Eur J Neurosci 25:603-610. CrossRef Medline

Sokoliuk R, VanRullen R (2013) The flickering wheel illusion: when alpha rhythms make a static wheel flicker. J Neurosci 33:1349813504. CrossRef Medline

Troncoso XG, Macknik SL, Otero-Millan J, Martinez-Conde S (2008) Microsaccades drive illusory motion in the enigma illusion. Proc Natl Acad Sci USA 105:16033-16038. CrossRef Medline

VanRullen R, Koch C (2003) Is perception discrete or continuous? Trends Cogn Sci 7:207213. CrossRef Medline

VanRullen R, Macdonald JS (2012) Perceptual echoes at $10 \mathrm{~Hz}$ in the human brain. Curr Biol 22:995-999. CrossRef Medline

VanRullen R, Busch NA, Drewes J, Dubois J (2011) Ongoing EEG phase as a trial-by-trial predictor of perceptual and attentional variability. Front Psychol 2:60. CrossRef Medline 